\title{
Política Nacional de Promoção da Saúde: um estudo de avaliabilidade em uma região de saúde no Brasil
}

\author{
The National Health Promotion Policy: an evaluability assessment \\ in a health region in Brazil
}

\author{
Maria Socorro de Araújo Dias ${ }^{1}$ \\ Irlanda Pontes de Oliveira ${ }^{2}$ \\ Lucilane Maria Sales da Silva ${ }^{3}$ \\ Maristela Inês Osawa Vasconcelos ${ }^{4}$ \\ Maria de Fátima Antero Sousa Machado ${ }^{5}$ \\ Franklin Delano Soares Forte ${ }^{6}$ \\ Lielma Carla Chagas da Silva ${ }^{7}$
}

\footnotetext{
${ }^{1}$ Departamento de Enfermagem, Centro de Ciências Biológicas e da Saúde, Universidade Regional do Cariri. R. Cel. Antonio Luis 1161, Pimenta. 63105000 Crato CE Brasil. socorroad@gmail.com ${ }^{2}$ Coordenadoria Regional de Saúde de Tianguá, Prefeitura Municipal de Ibiapina. Ibiapina CE Brasil.

${ }^{3}$ Centro de Ciências da Saúde, Universidade Estadual do Ceará. Fortaleza CE Brasil.

${ }^{4}$ Centro de Ciências da Saúde, Universidade Estadual Vale do Acaraú. Sobral CE Brasil. ${ }^{5}$ Departamento de Saúde, Centro de Ciências da Saúde, Universidade Regional do Cariri. Crato CE Brasil.

${ }^{6}$ Departamento de Clínica e Odontologia Social, Centro de Ciências da Saúde, Universidade Federal da Paraíba. João Pessoa PB Brasil.

${ }^{7}$ Núcleo de Estudos e Pesquisa em Saúde, Escola de Formação em Saúde da Família Visconde de Sabóia. Sobral CE Brasil.
}

\begin{abstract}
The National Health Promotion Policy (PNPS) is based on the broadened concept of health and presents the promotion of health as a set of strategies and ways of promoting health, both individually and collectively with responsibilities for the three federated entities. The scope of this study was to develop an evaluability assessment of the PNPS. It is a qualitative-based evaluability assessment conducted in a health region in the Brazilian Northeast in the year 2013. Participants in the study were 18 key informants directly involved in PNPS implementation, of which eight are municipal health secretaries, six are coordinators of primary health care, two are coordinators of epidemiological surveillance, a general regional coordinator and a regional primary care coordinator. The semi-structured interview and the legal documents of the policy were used for gathering information from 2006 to 2012. The construction of the logical model made it possible to reflect on the possible influences of the context on the PNPS implementation. The results showed that PNPS is not yet sufficiently established in the health region and pointed to the need to incorporate specific monitoring and evaluation instruments for this policy.
\end{abstract}

Key words Public policy, Evaluability, Health promotion
Resumo A Política Nacional de Promoção da Saúde (PNPS) baseia-se no conceito ampliado de saúde e apresenta sua promoção como um conjunto de estratégias e formas de produzir saúde, no âmbito individual e coletivo com responsabilidades para os três entes federados. Trata-se de estudo de avaliabilidade com abordagem qualitativa, realizada em uma região de saúde no nordeste brasileiro no ano de 2013. Participaram do estudo 18 informantes-chave envolvidos diretamente com a execução da PNPS, sendo que oito são secretários municipais de saúde, seis são coordenadores da atenção básica, dois coordenadores da vigilância epidemiológica, um coordenador regional geral e um coordenador regional da atenção básica. Foram utilizadas para a coleta de informações a entrevista semiestrutrurada e os documentos legais da política no período de 2006 a 2012. A construção do modelo lógico permitiu refletir sobre as possíveis influências do contexto sobre a implementação da PNPS. Os resultados demonstraram que a PNPS ainda não está suficientemente implantada na região de saúde e sinalizaram para a necessidade de incorporação de instrumentos de monitoramento e avaliação específicos para esta política.

Palavras-chave Políticas Públicas, Avaliabilidade, Promoção de Saúde 


\section{Introdução}

Durante a década de 1960, vários países realizaram debate sobre a determinação econômica e social da saúde, processo que contribuiu na perspectiva de uma abordagem positiva da saúde, cujo objetivo era superar a orientação predominantemente centrada no controle da enfermidade. Um dos movimentos históricos que contribuiu para a reorientação da abordagem à saúde foi o Movimento de Promoção à Saúde no Canadá. Este evento motivou a produção do documento, mais conhecido como Informe Lalonde "Uma Nova Perspectiva na Saúde dos Canadenses", lançado em 1974, com a caracterização do campo da saúde e a evidência de que o tradicional padrão assistencial tinha menos efeito para promover uma melhor saúde ${ }^{1}$.

A partir destes movimentos foi desencadeada uma série de iniciativas que se configuraram em conferências internacionais de promoção da saúde, que resultou em significativos produtos: Carta de Ottawa (Canadá, 1986), Declaração de Adelaide (Austrália, 1988); Declaração de Sundsvall (Suécia, 1991); Declaração de Bogotá (Colômbia, 1992); Declaração de Jacarta (Indonésia, 1997), Relatório da Conferência do México (2000); Carta de Bangkok (Tailândia, 2005)², Carta de Nairobi (Kenya, 2009)3 e Declaração de Helsinque sobre Saúde em Todas as Políticas (Helsinki, 2013)4.

No Brasil, desde meados da década de 1980, vários acontecimentos contribuíram para que a promoção da saúde fosse incorporada como uma nova filosofia na prática das políticas de saúde ${ }^{3}$. Dentre eles a realização da $8^{\text {a }}$. Conferência Nacional de Saúde (CNS), cujo tema foi "Democracia é Saúde". Esse espaço passou a ser um fórum de luta pela descentralização do sistema de saúde e pela implantação de políticas sociais que defendem e cuidam da vida das pessoas ${ }^{5}$. O Movimento da Reforma Sanitária Brasileira sofreu as mesmas influências sociais e históricas da construção da promoção da saúde em nível internacional ${ }^{6}$. Para Campos et al. ${ }^{7}$, esse movimento delineou a promoção da saúde como política que deve ser apresentada em todos os níveis de complexidade na gestão e na atenção do sistema de saúde.

Na Constituição Federal de 1988, o Estado brasileiro assumiu como seus objetivos precípuos a redução das desigualdades sociais e regionais. A Reforma Constitucional que criou o Sistema Único de Saúde (SUS) transferiu do nível federal aos municípios a responsabilidade de programar, atribuir os recursos, gerenciar, coordenar e avaliar os serviços de saúde oferecidos ${ }^{8}$.
A partir das definições constitucionais, da legislação que regulamenta o SUS, das deliberações das conferências nacionais de saúde, o Ministério da Saúde propõe um movimento indutor para definição da Política Nacional de Promoção da Saúde - PNPS, representando um esforço para o enfrentamento dos desafios de produção da saúde num cenário sócio-histórico cada vez mais complexo ${ }^{8,9}$.

Para Castro et al. ${ }^{3}$, a construção da PNPS no Brasil implicou num processo amplo de discussão com todas as áreas do MS, envolvendo gestores locais do SUS e universidades e um intenso processo de escuta dos diferentes atores sociais envolvidos. Esse processo ocorreu em duas etapas, sendo a primeira coordenada pela Secretaria Executiva do Ministério da Saúde durante a gestão de 2004-2004, e a segunda, entre 2005 e 2006, já a partir da Secretaria de Vigilância em Saúde ${ }^{10}$.

A culminância deste processo se dá com a institucionalização da PNPS, por meio da portaria MS/GM no 687/2006, a qual se propõe a ser uma política transversal com vistas a favorecer o diálogo entre os diversos setores do governo, setores privados e sociedade, compondo redes de compromisso e corresponsabilidade quanto à qualidade de vida da população ${ }^{11}$. Em 2014, o Ministério da Saúde propôs a revisão da PNPS. Para tal, visando garantir a capilaridade da participação nas cinco macrorregiões brasileiras na revisão da PNPS e favorecer a escuta dos diferentes segmentos envolvidos com o desenvolvimento e avaliação de ações de promoção da saúde no Brasil, foi firmado parceria entre a coordenação da PNPS e o Grupo Temático de Promoção da Saúde da Associação Brasileira de Saúde Coletiva - GT Promoção da Saúde e Determinantes Sociais Abrasco, com mediação da Organização Pan-Americana da Saúde - OPAS ${ }^{11,12}$.

Ainda neste contexto, múltiplos movimentos simultâneos nas diversas regiões do País foram utilizados como estratégia de escuta, mobilização e produção de sínteses que permitiram a elaboração coletiva da minuta da PNPS, com o propósito de ampla revisão e debate no âmbito do Comitê Gestor da Política ${ }^{10}$.

Diante do exposto, entende-se que descentralização da PNPS visa fortalecer na região de saúde a corresponsabilização dos municípios na implantação e implementação da PNPS no sistema local e regional de saúde, com vistas a melhoria da qualidade de vida da população. Desse modo, a PNPS tem potencial para se transformar num marco na história do SUS ${ }^{13}$. Considerando esta relevância, tornou-se oportuno realizar um 
estudo de avaliabilidade da PNPS numa região de saúde, a fim de elucidar como está o processo de efetivação desta, seus fatores dificultadores e facilitadores. Espera-se que os resultados advindos da avaliabilidade da PNPS servirão para uma futura avaliação quanto a sua real efetividade.

Nessa perspectiva, este artigo teve como objetivo realizar uma análise da avaliabilidade da política nacional de promoção da saúde numa região de saúde do nordeste brasileiro.

\section{Metodologia}

Trata-se de um estudo de avaliabilidade com abordagem qualitativa, o qual permite saber em que medida um programa/política está em condições de ser submetido a uma avaliação. Portanto, precede a etapa de avaliação propriamente $\operatorname{dita}^{14}$.

Quanto aos procedimentos éticos, esse estudo seguiu o que estabelece a resolução no 466/2012 do Conselho Nacional de Saúde, tendo sido recomendado pelo Comitê de Ética em Pesquisa da Universidade Estadual Vale do Acaraú-UVA.

Dentre os referenciais de avaliabilidade elegeu-se o proposto por Leviton et. al. ${ }^{15}$. Este autor considera que um estudo de avaliabilidade é um processo cíclico e dinâmico que consiste em: a) esclarecimentos sobre os objetivos e metas do programa; b) desenvolvimento e pactuação do modelo lógico com os interessados; c) análise e comparação entre a realidade da política e o modelo lógico; d) elaboração de recomendações.

O estudo foi realizado numa região de saúde que possui uma população de 298.017 habitantes ${ }^{16}$. A região é composta por oito municípios, sendo todos contemplados no estudo.

Inicialmente, foi realizada uma análise dos dados e informações de 2006 a dezembro de 2012. Este período foi selecionado, pois nele transcorreram as ações específicas da PNPS desde a sua publicação até a data de início da pesquisa. Foram tomados para análise os documentos legais que regem a PNPS que permitiu a aproximação e o entendimento de como funciona.

Nessa etapa inicial, visando destacar dados relevantes e estabelecer apropriações sobre a PNPS, foi realizada uma leitura flutuante dos seguintes documentos: 1. Portaria no 687/GM/ MS, de 2006, que aprova a Política Nacional de Promoção da Saúde (PNPS), estabelecendo as bases da atuação do Estado Brasileiro no campo da promoção da saúde; 2. Decreto no 6.286, de
2007, que institui no âmbito dos Ministérios da Educação e da Saúde, o Programa Saúde na Escola - PSE, com finalidade de contribuir para a formação integral dos estudantes da rede pública de educação básica, por meio de ações de prevenção, promoção e atenção à saúde; 3. Portaria no 1.409/GM/MS, de 2007, que instituiu o Comitê Gestor da Política Nacional de Promoção da Saúde - CGPNPS, com as atribuições de consolidar a implementação da Política Nacional de Promoção da Saúde e a Agenda Nacional de Promoção da Saúde; 4. Portaria GM/MS no 719, de 2011, que institui o Programa Academia da Saúde no âmbito do Sistema Único de Saúde, a ser implantado pelas Secretarias de Saúde do Distrito Federal e dos Municípios, com o apoio técnico das Secretarias Estaduais de Saúde e do Ministério da Saúde. Após este processo, os textos foram relidos, sendo destacadas as informações relevantes para a descrição da PNPS e a contribuição para a construção do modelo lógico. Nesta análise, foi possível identificar os objetivos da PNPS, as ações específicas e estratégicas de implantação para o seu desenvolvimento e implementação.

$\mathrm{Na}$ segunda etapa foi desenvolvido o modelo lógico e analisado com o propósito de descrever de forma clara e coerente o modo de funcionamento da PNPS, fundamentada nos documentos disponíveis sobre a PNPS no MS, já analisados na etapa inicial, e documentos da gestão municipal: Programação Anual de Saúde (PAS) e Relatório Anual de Gestão (RAG).

Concomitantemente foram realizadas as entrevistas com os informantes-chave. Participaram do estudo 18 informantes-chave envolvidos diretamente com a execução da PNPS, sendo que, destes sujeitos, oito são secretários municipais de saúde, seis são coordenadores da atenção básica, dois são coordenadores da vigilância epidemiológica, um, coordenador regional geral e, um, coordenador regional da atenção básica.

As entrevistas foram registradas em áudio e, posteriormente, foram transcritas, e, na sequência, realizada a leitura-análise exaustiva das transcrições, de forma a extrair informações quanto à concepção dos gestores em termos de formação/ função/tempo de atuação, percepção de objetivos, responsabilidades, recursos e resultados da PNPS. Em seguida, foi realizada a análise quanto à clareza e adequação dos objetivos e metas dos documentos em relação a PNPS, a fim de identificar possibilidades de superação de fragilidades e sugestões de inclusões. Na quarta etapa foram propostas recomendações. 


\section{Resultados e discussão}

Tomando em consideração a transversalidade da promoção da saúde, foi identificada a interface da PNPS com as demais políticas, programas e projetos vinculados à política nacional de saúde, embora se reconheça que o campo da promoção da saúde não tem seus limites contornados no setor saúde. Na Figura 1 são apresentadas estas conexões.

A Figura 1 nos conduz à interpretação de que as interfaces da PNPS com outras políticas e/ou projetos estratégicos da política nacional de saúde se complementam fundamentalmente. Ao tempo em que este dado pode evidenciar uma clara priorização da política de saúde trabalhar com problemas emergentes; visto que estes se encontram presentes tanto em iniciativas específicas quanto em políticas mais abrangentes; também é possível problematizar se esta direção não con- tribui com uma visão reducionista da promoção da saúde e, consequentemente, da própria PNPS, com ênfase em mudanças comportamentais. A promoção da saúde apresenta duas tendências de abordagens principais, conforme descrito por Buss ${ }^{17}$ : uma com enfoque comportamental, guiada pelos hábitos e estilos de vida e, outra, guiada pela concepção de qualidade de vida e pelos determinantes gerais das condições de vida e saúde da sociedade.

Outra leitura que a Figura 1 nos permite é a identificação de interfaces da PNPS com documentos de gestão orientadores da política nacional de saúde. Neste sentido, destaca-se o pacto pela saúde e, mais recentemente, o Contrato Organizativo de Ação Pública da Saúde.

Neste ínterim de apresentar a PNPS e correlacioná-la com os demais movimentos e processos da política nacional de saúde, registramos que, passados seis anos da institucionalização da

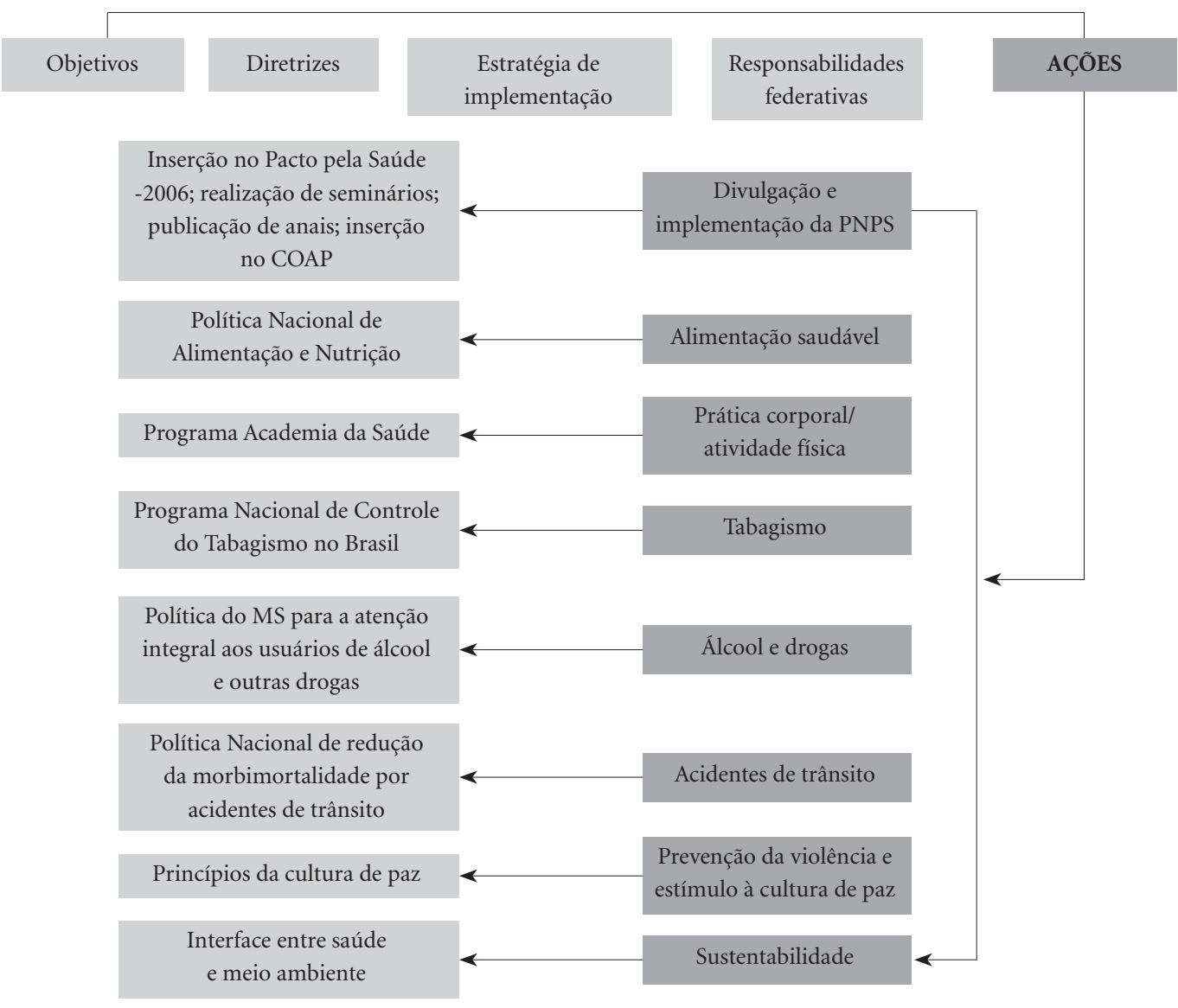

Figura 1. Interface da Política Nacional de Promoção da Saúde (Portaria no 687 MS/GM, de 30 de março de 2006) com demais programas e projetos do SUS. 
PNPS, temos a regulamentação pelo Poder Executivo Federal da lei no 8.080 , por meio do decreto $n^{\circ} 7.508$, de 2011 , visando dar transparência à gestão do SUS, por intermédio do contrato organizativo da ação pública da saúde, que prevê em suas metas como indicador universal: proporção de unidades de saúde com serviço de notificação de violência implantada; como indicador específico, a proporção de polos do Programa Academia da Saúde com profissional de saúde vinculado, e a proporção de municípios da região com ações ou políticas de promoção de equidade contempladas nos respectivos planos de saúde.

Identifica-se nestes indicadores uma relação com as ações específicas da PNPS. Situação que reforça a centralidade das ações específicas no modus operandi da referida política. Este olhar, de algum modo "reducionista" da PNPS, é problematizado nos movimentos de revisão a que esta foi submetida. Do final de 2013 ao início de 2014, a PNPS passou por um processo de revisão. Em texto preliminar para a reedição da PNPS, objeto de estudo e discussão no Seminário $\mathrm{Na}$ cional de revisão da PNPS, ocorridos nos dias 15 e 16 de maio de 2014, foi argumentado que nos últimos anos o MS assumiu novas demandas e compromissos nacionais e internacionais que o impulsionaram a um processo de revisão da PNPS; com destaque para as mudanças na legislação, como a publicação do decreto no 7.508 , de 2011 e da lei complementar no 141 , de $2012^{18}$ e publicação de Brasil (2014) $)^{12}$.

No âmbito internacional, eventos importantes promovidos pela Organização das Nações Unidas - ONU e suas agências, cujas Declarações foram ratificadas pelo governo brasileiro, também trouxeram desafios e propostas a serem consideradas nas políticas públicas, em particular para a PNPS ${ }^{8}$.

A estrutura prevista para a "nova” PNPS é orientada por princípios e valores que representam o fundamental de todas as práticas e ações na esfera de atuação da PNPS; objetivos que sinalizam a imagem objetivo; diretrizes, compreendidas como linhas que fundamentam as ações e explicitam as finalidades da PNPS; eixos transversais enquanto referências para a formulação da agenda de promoção da saúde, seguidos das estratégias operacionais e temas prioritários para a operacionalização da PNPS ${ }^{19}$.

A expectativa é que a nova versão da PNPS amplie as possibilidades de ações em promoção da saúde fundada nos determinantes da saúde, e que sejam tomados em consideração os contextos territoriais e a capacidade inventiva destes como respostas às suas necessidades e desejos.

Considerando que a PNPS vigente no curso da coleta de dados orientava-se por ações específicas, verificou-se a presença destas nos instrumentos de gestão (PAS e RAG) dos oito estudados no período da coleta de dados. Ao todo foram analisados 48 instrumentos de gestão dos municípios da região de saúde. Constatou-se que cinco dos oito municípios pactuaram ações na PNPS na PAS, e faziam referências a algumas destas ações no RAG. Estas ações se alinhavam ao eixo da atenção integral à saúde e/ou vigilância à saúde.

O exposto conduz ao entendimento de que a PNPS está normativamente bem consolidada, sendo um avanço verificado no desenvolvimento institucional do Estado brasileiro. Entretanto, a simples edição de normas legais não é condição suficiente para sua efetivação, daí que os desdobramentos práticos destas normas devem ser concretizados por ações e serviços públicos de promoção da saúde em todo o país, razão pela qual o acompanhamento vigilante da sociedade brasileira sobre o cumprimento das normas jurídicas editadas representa um fator importante para o desenvolvimento do Brasil na área de promoção da saúde ${ }^{20}$.

No que concerne à compreensão dos gestores acerca da PNPS, apresenta-se no Quadro 1 a relação entre os objetivos expressos na PNPS ${ }^{8}$ e os objetivos apreendidos por estes, a partir de um dos questionamentos feitos na entrevista: Quais os objetivos da PNPS?

Os gestores apresentaram conhecimento parcial acerca da intencionalidade da PNPS, sem, contudo, reproduzir literalmente os objetivos. De um objetivo geral e 12 objetivos específicos expressos na PNPS, foi possível estabelecer correspondência entre os objetivos manifestados pelos gestores no objetivo geral e em quatro específicos.

O objetivo geral denota a imagem-objetiva de um processo; com isso, é válido destacar o entendimento de alguns gestores que a PNPS se configura como uma possibilidade de contribuição para uma mudança paradigmática do modelo assistencial. Destaca-se, ainda, que foi apontado por estes a perspectiva da PNPS contribuir para a melhoria da qualidade de vida e ação sobre as vulnerabilidades. Este resultado denota que os gestores reconhecem a ação dos determinantes da saúde na promoção da saúde da população.

No que concerne à correspondência aos objetivos específicos, entendendo-os como orientadores do modus operandi, portanto, fundamental para a gestão em saúde, identifica-se uma inci- 
Quadro 1. Comparação entre os objetivos da PNPS e os objetivos citados pelos gestores da região de saúde estudada, 2014.

\begin{tabular}{|c|c|}
\hline Objetivos da PNPS & Objetivos apontados pelos gestores \\
\hline $\begin{array}{l}\text { 1. Promover a qualidade de vida e reduzir as } \\
\text { vulnerabilidades e os riscos à saúde, relacionados aos } \\
\text { seus determinantes e condicionantes }\end{array}$ & $\begin{array}{l}\text { - Melhorar a questão da qualidade de vida da } \\
\text { população } \\
\text { - Melhorar a qualidade de vida dos usuários } \\
\text { - Promover a qualidade de vida e reduzir as } \\
\text { vulnerabilidades e os riscos à saúde } \\
\text { - Melhor qualidade de vida para a população }\end{array}$ \\
\hline 2. Incorporar ações de promoção da saúde na $A B$ & $\begin{array}{l}\text { - Promover a política de trabalho da promoção à } \\
\text { saúde nas unidades básicas }\end{array}$ \\
\hline $\begin{array}{l}\text { 3. Ampliar a autonomia e a corresponsabilidade de } \\
\text { sujeitos, coletividades e poder público para minimizar } \\
\text { e/ou extinguir as desigualdades }\end{array}$ & $\begin{array}{l}\text { - Levar informação ao nosso usuário } \\
\text { - Estimular a população a cuidar da sua saúde por } \\
\text { meio das ações propostas pela política } \\
\text { - Orientação e educação da população em relação a } \\
\text { sua parcela de participação na saúde }\end{array}$ \\
\hline $\begin{array}{l}\text { 4. Promover entendimento da concepção ampliada de } \\
\text { saúde entre seus trabalhadores }\end{array}$ & - Não relatado pelos gestores \\
\hline $\begin{array}{l}\text { 5. Contribuir para a resolubilidade do sistema } \\
\text { (qualidade - eficácia - eficiência - segurança das ações } \\
\text { de PS) }\end{array}$ & $\begin{array}{l}\text { - Mudar o paradigma nos modelos assistenciais e } \\
\text { curativos }\end{array}$ \\
\hline $\begin{array}{l}\text { 6. Estimular alternativas inovadoras e socialmente } \\
\text { inclusivas das ações de PS }\end{array}$ & - Não relatado pelos gestores \\
\hline $\begin{array}{l}\text { 7. Valorizar o uso de espaços públicos para o } \\
\text { desenvolvimento das ações de PS }\end{array}$ & - Não relatado pelos gestores \\
\hline $\begin{array}{l}\text { 8. Favorecer a preservação do meio ambiente e a } \\
\text { promoção de ambientes mais seguros e saudáveis }\end{array}$ & - Não relatado pelos gestores \\
\hline $\begin{array}{l}\text { 9. Contribuir na elaboração de políticas públicas } \\
\text { integradas para a melhoria da qualidade de vida }\end{array}$ & - Não relatado pelos gestores \\
\hline $\begin{array}{l}\text { 10. Ampliar os processos de integração, com base na } \\
\text { cooperação, solidariedade e gestão democrática }\end{array}$ & - Não relatado pelos gestores \\
\hline $\begin{array}{l}\text { 11. Prevenir fatores determinantes e/ou condicionantes } \\
\text { de doenças e agravos }\end{array}$ & $\begin{array}{l}\text { - Promover a saúde e prevenir as doenças; } \\
\text { - Esclarecer a população e alertar para as ações de } \\
\text { prevenção }\end{array}$ \\
\hline 12. Estimular a adoção de modos de viver não violentos & - Adotar hábitos saudáveis de vida \\
\hline 13. Desenvolver uma cultura de paz no país & - Não relatado pelos gestores \\
\hline
\end{tabular}

Fonte: Elaborado pelos autores.

piência quanto ao conhecimento dos gestores, sendo os objetivos referidos aqueles mais relacionados diretamente à incorporação da promoção da saúde na atenção básica, na ampliação da autonomia e corresponsabilização, na prevenção de fatores determinantes e ou condicionantes de agravos e na adoção de modos de viver não violentos.

Desta forma, valores orientadores da Promoção da Saúde e que dialogam com o conceito lato de saúde, tais como ações inovadoras e inclusivas, preservação do meio ambiente, promoção de ambientes seguros e saudáveis, gestão democrática e cultura de paz, integração de políticas e solidariedade, que não foram mencionados pelos gestores, conduzem ao entendimento exposto por Buss ${ }^{17}$, de que a moderna promoção da saúde é guiada pela concepção de qualidade de vida e direcionada pelos determinantes gerais das condições de vida e saúde. E nesta perspectiva as ações estão voltadas para o coletivo de indivíduos e ao ambiente.

Ao tempo em que os objetivos revelam a imagem objetivo, os indicadores representam sinalizações de monitoramento num processo de gestão. Orientados por este entendimento, consultamos os gestores sobre os indicadores que norteavam a implementação da PNPS nos 
municípios sobre suas responsabilidades. Dos 18 gestores ouvidos, oito afirmaram não existir indicador de monitoramento no município relacionado à PNPS, dois reconheciam a existência, porém não recordavam quais eram. Os demais (08) afirmaram existir indicador e os relacionaram. Os indicadores expressos por estes estavam predominantemente vinculados ao Programa Saúde na Escola (PSE) e, assim, eram citados pelos gestores: "têm os indicadores do PSE"; foram citados também indicadores relativos à atividade física, redução da morbimortalidade por acidente e prevenção da violência.

$\mathrm{O}$ exposto demonstra que o PSE tem se incorporado nas agendas locais, sendo relacionado com a promoção da saúde. Entretanto não há indicadores, colocados pelos gestores, de sua contribuição para a implementação da PNPS.

Os discursos dos gestores acerca de suas responsabilidades aproximam das responsabilidades dos gestores descritas na PNPS. Estes apresentaram como responsabilidades, da coordenadoria da região de saúde, "[...] implementar, orientar, monitorar, incrementar a política na região, bem como trabalhar a implementação junto às equipes de saúde da família”. Vale ressaltar que a coordenadoria regional representa a secretaria de saúde do Estado na região de saúde, portanto, assume na região as responsabilidades do Estado para a implementação da política descrita ${ }^{21}$. Para os secretários de saúde e coordenadores da atenção básica e vigilância epidemiológica, foi identificado como responsabilidade, segundo fala dos gestores: “[...] promover e executar as atividades que estão previstas na PNPS, garantir os recursos, fazer com que as ações definidas no plano sejam cumpridas, montar uma estrutura com recursos humanos e materiais para facilitar o trabalho, apoiar a equipe de atenção básica, secundária, tanto com suporte técnico como financeiro, pactuar e alocar recursos financeiros para implementação da PNPS”. E “supervisionar as ESF quanto às atividades de promoção, de prevenção à saúde, trabalhar o projeto de vigilância, prevenção de violência e acidentes, incentivar as equipes a trabalhar, e cobrar as ações, organizar e planejar os processos educativos, acompanhar os indicadores".

Diante do exposto, as responsabilidades descritas pelos gestores constituem-se em reponsabilidades gerais de incumbência dos gestores em processos de implementação de políticas públicas, não evidenciando singularidades de responsabilidades que guardam mais proximidades com a PNPS, a exemplo da articulação intersetorial, a partir do estabelecimento de parcerias e articulação e apoio às experiências de educação popular, desenvolvimento de processos de educação permanente, dentre outras. Chama-se a atenção para ações específicas do ente estadual e que não foram reconhecidas por estes, situação que denota a necessidade de alinhamento entre as responsabilidades assumidas e as responsabilidades incorporadas pelos gestores.

Igualmente, quando comparamos as responsabilidades inerentes ao ente municipal, expressas na PNPS, com as responsabilidades manifestadas pelos gestores municipais, identifica-se que estes evidenciaram responsabilidades que comungam mais proximamente com as descritas na PNPS. Entretanto, mais uma vez, responsabilidades que expressam a uma identidade forte da PNPS não foram apontadas pelos gestores municipais, a intersetorialidade e reforço da ação comunitária.

O reconhecimento e cumprimento das responsabilidades de implementação da PNPS pelos três entes federativos contribuiria para a execução da referida política. Para Traverso-Yépez ${ }^{22}$, as orientações para a implementação da PNPS, na sua maioria, estão voltadas para os gestores. Situação que denota, segundo a autora e com a qual se concorda, a necessidade expressa de qualificar esse segmento de profissionais, configurando assim uma possibilidade de fortalecimento da promoção da saúde no campo da atenção básica, mais especificamente na ESF.

Outrossim, entendemos que, embora a PNPS evidencie com clareza as responsabilidades referentes aos três entes federados, a implementação de uma política não limita-se ao âmbito da gestão. Para que uma política seja viva, faz-se necessário a participação ativa de trabalhadores e comunidade.

Dentre os recursos identificados pelos gestores como necessários à efetiva implementação da PNPS está a estrutura física. Entretanto, esta é considerada insuficiente para a realização das ações de promoção da saúde, embora um gestor municipal tenha relatado a melhora da estrutura requerida, a partir do Programa do Requalifica UBS, o qual se configura como uma iniciativa do ente federal denominado de Programa de Requalificação de Unidades Básicas de Saúde (UBS), nos componentes reforma, ampliação e construção, onde os municípios cadastram as propostas no Sistema de Monitoramento das Obras - Sismob, e, se aprovadas, são publicadas por meio de portarias federais ${ }^{23}$.

Também foi referido pelos gestores que os recursos financeiros destinados a PNPS são parcos, 
situação reafirmada pela insuficiência de material de apoio às atividades. Reafirma-se que o financiamento de uma política não deve ficar unicamente sobre a égide de um ente financiador.

O capital humano tratado pelos gestores como recurso, como podemos perceber no discurso "[...] um recurso necessário para implementação da PNSP é a contratação de mais profissionais, um profissional habilitado para auxiliar a ESF, é necessário mais recurso humano". Identificado como algo necessário, de certa forma ainda insuficiente. Além de requerer maior capilaridade de ações de educação permanente. Ressalta-se que a educação permanente como foco na promoção da saúde é uma das responsabilidades da componente gestão na PNPS. Gestores também compreenderam que fazem parte dos recursos necessários para a PNPS, a partir da compreensão e da sensibilização destes para efetiva implementação, embora um deles não compreenda dessa forma.

Vale ressaltar que não se pode desconsiderar a importância de ambientes adequados com infraestrutura e materiais de apoio para ações promotoras de saúde, pois a essência da promoção da saúde está no diálogo com o outro (outra pessoa, outro setor, outra prática). Não se constitui, portanto, situação sine qua non, a existência de infraestrutura para a realização de ações de promoção da saúde, visto que o espaço da promoção da saúde é o espaço onde a vida acontece, portanto, as ações devem ocorrer predominantemente no território.

O Quadro 2 sinaliza os fatores que contribuíram e outros que configuraram como dificultadores, para a execução da PNPS. Para alguns gestores, a relação que as secretarias de saúde possuem com as demais secretarias permitem o exercício da intersetorialidade, fundamento da promoção da saúde. Os gestores expressam ainda que a existência de profissionais capacitados, a cobertura da ESF e a implementação do Núcleo de Apoio à Saúde da Família - NASF; convênios com instituições de ensino, adesão da população às ações que são implementadas, principalmente em atividades físicas, como também adesão dos profissionais; contratação de profissional específico para a PNPS e compromisso dos profissionais são expressões de potencialidades para execução da PNPS.

Com relação às situações limites ou dificuldades, mais uma vez, emergiu o componente financeiro, seja para implementação de ações, seja para a capacitação dos profissionais. Merece ressaltar ainda dificuldades citadas relatadas como: falta de estrutura na atenção básica; ausência de cobrança da aplicação dos recursos; a falta de avaliação da política; escassez de recursos humanos; dificuldade de transportes; falta de adesão da população; burocratização dos processos de trabalho, sobrecarga de atividades para atenção básica; desconhecimento da PNPS por trabalhadores e população. É importante relatar que algumas situações ora emergem como potencialidade e ora como dificuldade, configurando movimentos e momentos diferentes vivenciados pelos gestores.

Já o Quadro 3 elucida os resultados reconhecidos pelos gestores municipais como oriundos da implementação da PNPS na região de saúde estudada.

Os resultados evidenciados pelos gestores municipais transitam pelas áreas prioritárias da PNPS, expressas nas ações específicas, principalmente naquelas relacionadas ao controle do tabagismo, incorporação de práticas de atividades físicas, influenciando, inclusive, o uso de medicamentos antidepressivos, além da diminuição no número de óbitos por acidentes de trânsito, influenciado por ações educativas que visam, entre outros objetivos, incentivar o uso de capacetes pelos motoqueiros (reduziu o número de mortes, mas aumentou o número de acidentes). Foi mencionada também a influência na PNPS na formação de grupos de diferentes faixas etárias, com o objetivo de desenvolver práticas promotoras de saúde. Os gestores sinalizaram ainda que a estratégia do NASF muito colaborou com a implementação da PNPS. Por fim, é referido por gestores que a PNPS cumpre seu papel maior ao apresentar como resultado a contribuição para melhoria da qualidade de vida das pessoas.

Tendo percorrido as etapas de exploração dos documentos oficiais relativos à institucionalização e implementação da PNPS e ouvido os gestores estratégicos desta na região de saúde estudada, seguiu-se orientado pelo referencial da avaliabilidade com a apresentação do modelo lógico da PNPS na região.

Baseando-se nesse contexto, foi realizada a construção do modelo lógico da PNPS. Este retratou um quadro que pode ser uma referência para ajudar a todos os envolvidos na implementação da PNPS. Desta forma, é criada uma possibilidade de rever a política, reforçando a necessidade de um processo participativo na revisão desta, ouvindo gestores e executores das ações nos territórios.

Como afirma Silva ${ }^{24}$, a teoria não é detalhada nos documentos oficiais, o que dificulta uma análise adequada. Segundo o autor, a partir da 
Quadro 2. Facilidades e dificuldades no processo de execução da PNPS. 2014.

\begin{tabular}{|c|c|c|}
\hline Municípios & Facilidades & Dificuldades \\
\hline $\mathrm{A}$ & $\begin{array}{l}\text { São as parcerias com as secretarias de } \\
\text { agricultura e meio ambiente, cultura, } \\
\text { esporte e educação; profissionais capacitados } \\
\text { (educação continuada em outros temas, } \\
\text { trabalho em grupos). }\end{array}$ & $\begin{array}{l}\text { A estrutura da atenção básica, equipamentos, } \\
\text { questão financeira específica para a PNPS, não há } \\
\text { cobrança da aplicação do recurso, profissionais da } \\
\text { atenção básica são muito sobrecarregados. }\end{array}$ \\
\hline B & $\begin{array}{l}\text { Uma rede disponível, número de } \\
\text { profissionais capacitados, apoio da gestão, a } \\
\text { intersetorialidade. }\end{array}$ & $\begin{array}{l}\text { Questão financeira para capacitar todo o corpo } \\
\text { profissional; falta de uma coordenação específica, } \\
\text { falta de avaliação da política, não divulgação } \\
\text { dessa política para a população. }\end{array}$ \\
\hline $\mathrm{C}$ & Equipe do NASF e cobertura da ESF. & $\begin{array}{l}\text { Falta de recursos para estruturar as UBS, muita } \\
\text { burocracia. }\end{array}$ \\
\hline $\mathrm{D}$ & $\begin{array}{l}\text { O próprio usuário, contratação de } \\
\text { profissional específico, as parcerias que nós } \\
\text { tivemos, guarda municipal, com as escolas, } \\
\text { com o conselho tutelar, com as equipes do } \\
\text { CRAS, do CREAS. }\end{array}$ & $\begin{array}{l}\text { Precisa de profissionais para atender no local } \\
\text { visitado, em cada localidade fazendo essas } \\
\text { ações acontecer, tinha só um transporte, seriam } \\
\text { necessários mais profissionais e mais transportes. }\end{array}$ \\
\hline $\mathrm{E}$ & $\begin{array}{l}\text { Apoio da administração, boa equipe de } \\
\text { saúde, cobertura superior a } 80 \% \text { da ESF, } \\
\text { bons convênios com Universidade Pública e } \\
\text { Privada, implementação do NASF, gestão do } \\
\text { coordenador da atenção básica. }\end{array}$ & $\begin{array}{l}\text { Questão cultural da população; algumas } \\
\text { categorias profissionais que ainda são dificultosas } \\
\text { de trabalhar a promoção da saúde em ir para } \\
\text { as escolas, de sair do consultório em si, para } \\
\text { desenvolver ações nas escolas, na comunidade. }\end{array}$ \\
\hline $\mathrm{F}$ & $\begin{array}{l}\text { Integração que se tem num município } \\
\text { - todas as secretarias profissionais são } \\
\text { compromissadas, a sensibilidade do gestor. }\end{array}$ & $\begin{array}{l}\text { Escassez de recursos humanos e financeiros; são } \\
\text { várias as prioridades, e você termina sem saber o } \\
\text { que priorizar. }\end{array}$ \\
\hline G & $\begin{array}{l}\text { Pessoal que é do NASF apoio que a gente tem } \\
\text { tido do gestor maior, do prefeito. }\end{array}$ & $\begin{array}{l}\text { Ausência de material pelos processos de licitação; } \\
\text { problemas na licitação, licitação na compra de } \\
\text { materiais permanentes, licitação na questão das } \\
\text { gráficas. }\end{array}$ \\
\hline $\mathrm{H}$ & $\begin{array}{l}\text { Adesão da população às ações que } \\
\text { são implementadas, principalmente } \\
\text { em atividades físicas com adesão dos } \\
\text { profissionais; as parcerias. }\end{array}$ & $\begin{array}{l}\text { Ausência de espaços físicos adequados e ausência } \\
\text { de profissionais capacitados, necessidade de } \\
\text { capacitação específica para essa área; hábitos } \\
\text { culturais. }\end{array}$ \\
\hline
\end{tabular}

Fonte: Elaborado pelos autores.

análise da teoria de um programa, destaca-se a importância do modelo lógico para a identificação de deficiências ou problemas em seu fluxo, que poderão interferir no seu desempenho. Avaliar a qualidade da teoria significa verificar se o programa está bem desenhado e se apresenta um plano plausível de alcance dos resultados esperados, por isso, a pesquisa ouviu os gestores regionais e municipais, pois estes são diretamente responsáveis pela implementação da PNPS em seus territórios, conhecendo as potencialidade e limites que permeiam a realidade local.

A construção do modelo lógico (Figura 2) permitiu refletir sobre as possíveis influências do contexto sobre a implementação da PNPS. A partir da construção do modelo lógico foi possível inferir a sustentabilidade das hipóteses as- sumidas na sua estruturação de uma política e o alcance de resultados.

Considerando que o objetivo de um modelo lógico é ser um desenho funcional que se apresente visualmente como um programa/política que poderá se desenvolver, e sua possível execução em um determinado cenário e como pretende resolver os problemas iniciais identificados ${ }^{24}$, o modelo lógico apresentado teve como pretensão apresentar a implementação da PNPS na região de saúde estudada.

A PNPS constitui como já referida, enquanto estratégia de produção de saúde, ou seja, como um modo de pensar e de operar articulada às demais políticas do sistema de saúde, contribuindo na construção de ações que possibilitam responder às necessidades sociais em saúde. Retomando 


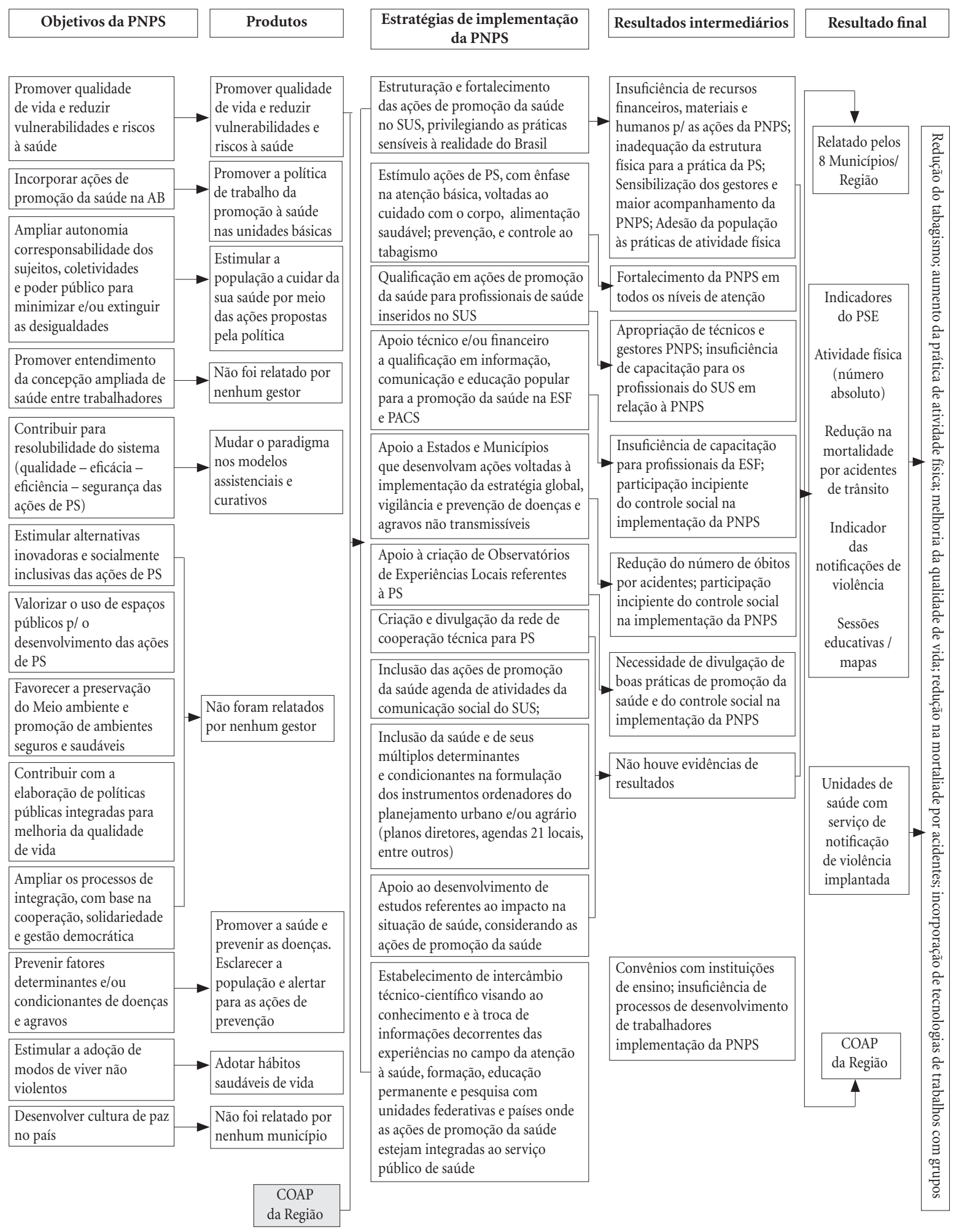

Figura 2. Modelo lógico da Política Nacional de Promoção da Saúde, 2014. 
a matriz da PNPS, apresentada no início desta discussão e colocada em diálogo com o modelo lógico da implementação da PNPS na região de saúde estudada, infere-se que a PNPS está em processo de implementação, com registros de alguns resultados, porém com um desejo expresso de ampliação de recursos materiais e imateriais, com vistas à consolidação deste processo. Destaca-se, ainda, uma intencionalidade dos gestores de implementar e incorporar a promoção da saúde nos processos de produção de saúde.

\section{Conclusões}

O estudo de avaliabilidade da PNPS contribuiu para um melhor entendimento do campo da promoção da saúde e os movimentos de institucionalização, implantação e implementação desta. O referencial teórico-metodológico que orientou este estudo de avaliabilidade nos ajudou a elaborar respostas aos questionamentos inicialmente postos e visualizar a exiguidade dos objetivos.

O recorte estudado numa região de saúde permitiu conhecer os desafios dos movimentos macro e micro vivenciados na implementação da PNPS, com destaque para a insuficiência de apropriação da PNPS por parte dos gestores; ao tempo que apresentam um desejo manifesto de potenciá-la.

A análise dos instrumentos de gestão permitiu também averiguar que não há indicadores para as ações de promoção da saúde nos municípios, situação que denota a insuficiência de instrumentos de monitoramento e avaliação, podendo contribuir para a invisibilidade de alguns resultados. Portanto, é possível compreender que há necessidade de se estabelecer indicadores de acompanhamento para as ações de promoção da saúde, devendo estes estar expressos nos instrumentos orientadores de gestão.

Na região de saúde estudada verificou-se que a PNPS ainda não está suficientemente implantada. A maioria dos municípios possui em seus instrumentos de gestão ações específicas da política, mas na prática ainda há muitas dificuldades para a efetivação desta.

Apresentam-se recomendações para a implementação da PNPS, tais como: realizar um diagnóstico de como a política acontece nos municípios brasileiros; investir na política em sua totalidade, não apenas em ações específicas; incluir mais indicadores da PNPS nas pactuações do SUS; expandir para todos os municípios os sistemas de informação que visam coletar informações para a avaliação desta; revisar periodicamente o modelo lógico da PNPS; disseminar os resultados desta pesquisa para gestores, técnicos e profissionais da estratégia da saúde da família; promover maior interação entre os objetivos, diretrizes e ações da PNPS com os gestores, profissionais da ESF, controle social; meio acadêmico e com a sociedade em geral; melhorar a divulgação e o financiamento acerca da PNPS.

Nesse sentido, é importante que a promoção da saúde no Brasil possa pensar em políticas públicas saudáveis voltadas para a diminuição das iniquidades sociais, evidenciadas nas desigualdades em saúde na vida individual e coletiva, visando alcançar o fortalecimento da ação comunitária necessária ao exercício da autonomia e valorizar o conhecimento popular e da participação social, já que estas estão na base da promoção da saúde.

Ressalta-se como limitação do estudo, que o resultado final apresentado no modelo lógico foi baseado no discurso dos gestores, pois não temos indicadores formalizados nos municípios para o monitoramento e avaliação da PNPS.

O modelo lógico construído nesse estudo pode ser estudado e revisado a qualquer momento, à medida que novas diretrizes, estratégias de implementação são inovadas ou que outras lentes analíticas e/ou avaliativas direcionem para a PNPS.

\section{Colaboradores}

MSA Dias, IP Oliveira, LMS Silva, MIO Vasconcelos, MFAS Machado, FDS Forte e LCC Silva participaram igualmente de todas as etapas de elaboração do artigo. 


\section{Referências}

1. Brasil. Ministério da Saúde (MS). As Cartas da Promoção da Saúde. Brasília: MS; 2002.

2. Lopes MSV, Saraiva KRO, Fernandes FC, Ximenes LB. Análise do Conceito de Promoção da Saúde. Text contexto enferm 2010; 19(3):461-468.

3. Castro AM, Sperandio AMG, Gosch CS, Rocha DG, Cruz DKA, Malta DC, Albuquerque GM, Zancan L, Westphal M, Neto OLM, Durán PRF, Sá RF, Mendes R, Moysés ST, Marcondes W, orgarnizadores. Curso de extensão para gestores do SUS em promoção da saúde. Brasília: CEAD/FUB; 2010.

4. World Health Organization (WHO). Health in all policies: Helsinki statement. Framework for country action. [acessado 2014 nov 6]. Disponível em: http://www. who.int/healthpromotion/conferences/8gchp

5. Brasil. Ministério da Saúde (MS). Conselho Nacional de Saúde. Relatório Final da $8^{a}$ Conferência Nacional de Saúde. 17 a 21 de mar. de 1986. Brasília: MS; 2006.

6. Andrade LOM, Barreto ICHC, Paula JB. Promoção da Saúde: aspectos históricos e conceituais. In: Catrib AMF, Dias MAS, Frota MA, organizadores. Promoção da Saúde no Contexto da Estratégia Saúde da Família. Campinas: Saberes; 2011.p. 23-44.

7. Campos GW, Barros RB, Castro AM. Avaliação da Política Nacional de Promoção da Saúde. Brasília, 2004. Cien Saude Colet 2004; 9(3):745-749.

8. Brasil. Ministério da Saúde (MS). Política Nacional de Promoção da Saúde. Brasília: MS; 2006.

9. Buss PM. Promoção da Saúde e qualidade de vida. Cien Saude Colet 2000; 5(1):163-177.

10. Rocha DG, Alexandre VP, Marcelo VC, Rezende R, Nogueira JD, Sá RF. Processo de revisão da Política Nacional de Promoção da Saúde: múltiplos movimentos simultâneos. Cien Saude Colet 2014; 19(11):4313-4322.

11. Brasil. Portaria no 340, de 04 de março de 2013. Redefine o Componente Construção do Programa de Requalificação de Unidades Básicas de Saúde (UBS). Diário Oficial da União 2013; 05 mar.

12. Brasil. Ministério da Saúde (MS). Política Nacional de Promoção da Saúde - PNaPS: revisão da Portaria MS/ GM nº 687, de 30 de março de 2006. Brasília: MS; 2014.

13. Rodrigues CR, Ribeiro KSQS. Promoção da saúde: a concepção dos profissionais de uma unidade de saúde da família. Rev Trabalho Educação e saúde 2012; 10(2):235-255.

14. Hartz ZMA, Champagne F, Contandriopou AP, Leal MC. Avaliação do programa materno-infantil: análise de implantação em sistemas locais de saúde no nordeste do Brasil In: Hartz ZMA, organizadores. Avaliação em Saúde: dos modelos conceituais à prática na análise da implantação de programas. Rio de Janeiro: Editora Fiocruz; 1997. p. 89-132.
15. Leviton LC, Collins CB, Laird BL, Kratt PP. Teaching Evaluation Using Evaluability Assessment. Thousands Oaks, London, New Delhi: Sage Publications; 1998.

16. Instituto Brasileiro de Geografia e Estatística (IBGE). 2011 nov [acessado 2011 nov 26]. Disponível em: http://www.ibge.gov.br/cidadesat/link.php?codmun $=230020$

17. Buss PM. Uma introdução ao Conceito de Promoção da Saúde. In: Czeresnia D, Freitas CM, organizadores. Promoção da Saúde: conceitos, reflexões, tendências. Rio de Janeiro: Fiocruz; 2009. p.19-42.

18. Brasil. Ministério da Saúde (MS). Anais: I Seminário sobre a Política Nacional de Promoção da Saúde. Brasília: MS; 2012. (Série D. Reuniões e Conferências).

19. Brasil. Ministério da Saúde (MS). Politica Nacional de Promoção da Saúde (versão preliminar). Brasília: MS; 2014.

20. Malta DC, Castro AM. Avanços e resultados na implementação da Política Nacional de Promoção da Saúde. Boletim técnico do SENAC 2009; 35(2):63-71.

21. Brasil. Ministério da Saúde (MS). Diretrizes operacionais dos Pactos pela Vida, em Defesa do SUS e de Gestão. Brasília: MS; 2006. (Série A. Normas e Manuais Técnicos).

22. Traverso-Yépez MA. Dilemas na promoção da saúde no Brasil: reflexões em torno da política nacional. Interface (Botucatu) 2007; 11(22):223-238.

23. Brasil. Portaria no 340, de 04 de março de 2013. Redefine o Componente Construção do Programa de Requalificação de Unidades Básicas de Saúde (UBS). Diário Oficial da União 2013; 5 mar.

24. Silva RC. A avaliabilidade do Programa SESI de prevenção de quedas na indústria de construção civil na Bahia [dissertação]. Salvador: Universidade Federal da Bahia; 2007.

Artigo apresentado em 06/07/2015

Aprovado em 13/01/2016

Versão final apresentada em 15/01/2016 